\title{
Blood Coagulation at Major Orthopaedic Surgery
}

Antropova IP', Reino EV ${ }^{1}$, Yushkov BG ${ }^{2,3}$, Shlykov IL ${ }^{1}$ and Varaksin $\mathrm{AN}^{4}$

${ }^{1}$ Ural Research Institute of Traumatology and Orthopedics, Russia

${ }^{2}$ Institute of Immunology and Physiology, RAS Ural Branch, Russia

${ }^{3}$ Ural Federal University, Russia

${ }^{4}$ Ural Federation University Institute of Industrial Ecology, RAS Ural Branch, Yekaterinburg, Russian Federation

\begin{abstract}
Thromboelastography (TEG) provides a means of monitoring coagulation postoperatively, the triggers involved are not clear. We studied the TEG parameters after total hip arthroplasty (THA), exploring the relationship with markers of hemostasis. In samples collected from 61 patients (33-72 years old), TEG parameters, global coagulation indices and specific hemostatic markers (TAT, D-dimer, and $\beta$-thromboglobulin) were assessed. Blood samples were taken before THA, 30 min after completion of surgery, and post-THA (Days 1, 3, 7, and 14). TAT levels peaked upon completion of THA, declining on Day 1 and normalizing by Day $3 ; \beta$-thromboglobulin levels increased sharply to peak upon completion of THA, returning to baseline levels after Day 7; and the TEG coagulation index $(\mathrm{Cl})$ of whole blood increased immediately after THA, peaked within 7 days, and remained elevated for up to 14 days. After surgery $(30 \mathrm{~min}), \mathrm{Cl}$ correlated significantly with platelet count $(r=0.31, p<0.05)$ and $\beta$-thromboglobulin level $(r=0.60, p<0.05)$. On postoperative Day $1, \mathrm{Cl}$ correlated with fibrinogen level $(r=0.42$, $p<0.05)$ and platelet count $(r=0.36, p<0.05)$. By Day 3 , a correlation between $\mathrm{Cl}$ and activity of the external coagulation pathway was noted. $\mathrm{Cl}$ and PT also correlated significantly on Day $7(r=-0.45, p<0.05)$ and Day 14 $(r=-0.47, p<0.05)$. D-dimer concentration increased sharply at completion of surgery, declined at Day 3 , rose again by Day 7, and remained elevated until Day14. On the other hand, the whole blood lysis index (Ly30) was unchanged at completion of surgery and during postoperative Day 1 , increased significantly on Day 3 , and then returned to baseline levels by Day 7 . In conclusion, thrombin formation after major orthopedic surgery declines; but the total coagulation potential of blood (under standard anticoagulant treatment) peaks within one week and remains elevated at least until postoperative Day 14, correlating with activity of the external coagulation pathway during this time.
\end{abstract}

Keywords: Coagulation, Thromboelastography, Total hip arthroplasty

\section{Introduction}

Since the days of Virchow, a hypercoagulable state has been recognized as a risk factor for the development of venous thromboembolic complications; and this concept is upheld by current research [1-4]. Deep vein thrombosis and pulmonary embolism still pose a real threat after major orthopedic surgery [5,6]. The prothrombotic state generated by such procedures (total large joint arthroplasty in particular) may be related to tissue factor expression, platelet activation, endothelial dysfunction, fibrinolytic disorders, and decreasing of the natural anticoagulants [7-10]. Anticoagulant therapy is thus widely applied as a preventative measure [2]. However, due to inherent difficulties in therapeutic drug monitoring, the use of anticoagulants is primarily a function of surgical risk group, with little attention paid to a patient's hemocoagulative state. In patients with acquired or hereditary thrombophilia or with a propensity for hemorrhagic manifestations, an individualized approach is needed to address coagulative imbalances.

Routine coagulation tests typically do not detect a hypercoagulable state. Instead, the kinetics of clot formation, clot strength, platelet function, and fibrinolysis may be determined by thromboelastography (TEG), which provides a global picture of hemostatic ability and the viscoelastic properties of clot $[11,12]$. This technique is useful for monitoring coagulation during and after surgical procedures, prompting some sources $[13,14]$ to advocate its use in the screening patients with suspected prothrombotic states. Unfortunately, the underpinnings of postoperative hypercoagulative conditions are not yet fully appreciated.

Herein, we report our findings on the dynamics of clot formation and lysis in whole blood after total hip arthroplasty, correlating measured parameters with specific hemostatic markers.

\section{Materials and Methods}

\section{Patients}

The study was approved by the local Ethical Committee and was carried out in accordance with the standards stipulated by the World Medical Association Declaration of Helsinki. All participants granted informed consent.

Blood samples were collected from 61 patients (age range, 33-72 years; mean age, $54 \pm 2$ years; males, 29 ; females, 32 ) with osteoarthritis who were admitted for primary total hip arthroplasty (THA). Patients were excluded on the following basis: (1) known cancer, (2) hepatic or renal dysfunction, (3) history of hematologic disorder, and (4) preoperative treatment with anti-aggregative or anticoagulant drugs. Samples were taken perioperatively (on admission to the hospital and $30 \mathrm{~min}$ after completion of surgery) and postoperatively (Days 1, 3, 7, and 14).

All subjects received low-molecular-weight heparin (enoxaparin)

*Corresponding author: Irina P Antropova, Senior Researcher, Federa State Institution, Ural Research Institute of Traumatology and Orthopedics, 7 Bankovsky alley, 620014, Yekaterinburg, Russia Federation, Tel: 7-9221105359; E-mail: aip.hemolab@mail.ru

Received October 03, 2013; Accepted November 22, 2013; Published November 25,2013

Citation: Antropova IP, Reino EV, Yushkov BG, Shlykov IL, Varaksin AN (2013) Blood Coagulation at Major Orthopaedic Surgery. J Blood Disorders Transf 5 : 178. doi: $10.4172 / 2155-9864.1000178$

Copyright: (c) 2013 Antropova IP, et al. This is an open-access article distributed under the terms of the Creative Commons Attribution License, which permits unrestricted use, distribution, and reproduction in any medium, provided the original author and source are credited. 
as thromboembolic prophylaxis. Injections $(40 \mathrm{mg})$ were given as follows: (1) 12 hours prior to surgery, (2) 12 hours post-arthroplasty, and (3) daily thereafter during study. To prevent excessive blood loss, tranexamic acid was administered perioperatively.

\section{Blood collection and storage}

Venous blood samples were drawn into collection tubes containing 0.129 M sodium citrate anticoagulant (Sarstedt AG \& Co, Germany), at a 1:9 ratio of anticoagulant to whole blood. Platelets were quantified using EDTA-stabilized blood. Citrated platelet-poor plasma, prepared by centrifugation $(15 \mathrm{~min}$ at $3000 \times \mathrm{g})$, was stored at $-20^{\circ} \mathrm{C}$ for later assay (ELISA).

\section{TEG analysis}

For TEG analysis, whole blood collected in 3.2\% trisodium citrate was analyzed within 30-90 min of collection via computerized TEG coagulation analyzer (TEG model 5000, Haemoscope Corp, Niles, IL, USA). The reaction time (R; normal range, 9-27 min) reflects the latency period for blood (once placed in the analyzer) to initiate fibrin formation. The alpha angle (Ang; normal range, 22-58 ${ }^{\circ}$ ) is the slope of the tracing, indicating the rapidity of fibrin build-up and cross-linking. The maximal amplitude (MA; normal range, 44-64 mm) is a direct function of the maximum dynamic properties of fibrin and platelet bonding and represents the ultimate strength of the fibrin clot; and percent lysis $30 \mathrm{~min}$ after MA is reached (Ly30; normal range 0-8\%) is a related parameter. The Coagulation Index (CI) is a measure of the patient's overall coagulation status (normally between -3 and +3 ).

\section{Hemostasis assay}

Prothrombin time (PT) and activated partial thromboplastin time (aPTT) were tested on the ACL-200 (Instrumentation Laboratory Co, Bedford, MA, USA), and fibrinogen (Clauss method) was quantified on the CA-50 (Sysmex Corp, Japan). Commercial ELISA assays were utilized for plasma concentrations of thrombin-antithrombin (TAT) complexes (Enzygnost TAT micro, Dade Behring Marburg GmbH, Marburg, Germany); D-dimer levels in plasma (Asserachrom D-dimer, Diagnostica Stago, France); and $\beta$-thromboglobulin (Asserachrom $\beta-T G$ ), as an index of platelet activation. Platelet counts were performed on EDTA-stabilized blood, using a Cell-Dyn-1700 hematologic analyzer (Abbott Diagnostics, Abbott Park, IL, USA).

All determinations were conducted according to protocols stipulated by the manufacturers.

\section{Statistical analysis}

Distributions of determinants were analyzed using the KolmogorovSmirnov test, expressing data as median and interquartile range (IQR). Friedman ANOVA and Wilcoxon tests were applied to detect pre- and post-surgical differences. Spearman's rank test was used for correlation analysis. Statistical significance was set at $\mathrm{p}<0.05$.

\section{Results}

After completion of surgery, enzymatic clot formation (R) accelerated significantly, with an observable trend toward normalization within one day. However, R-values continued to decline for up to 14 days, approaching baseline. Ang values increased after surgery; tended to normalize on Day 1; and by Day 7, again rose significantly and remained high (relatively to baseline) until Day 14. MA increased throughout postoperative Day 1 , peaked by Day 7 , and remained elevated until Day 14. The total blood hemostatic potential (TEG CI) increased immediately after THA, peaking on Day 7 , and remained elevated (relative to baseline) until Day 14. Whole blood lysis (Ly30) was unchanged at completion of surgery and during postoperative Dayl, showing a significant increase on Day 3 but returning to admission levels by Day 7 after THA (Table 1).

Factors involved in external pathway of coagulation fell at completion of surgery, whereas factors of the internal pathway dropped markedly by postoperative Day 1 . However, all factors were restored in 3 days. TAT concentrations peaked at completion of surgery, and then declined the next day, returning to baseline levels by Day 3 postoperatively. Maximum concentrations of fibrinogen were noted by Day 3 after THA, and level remained elevated until Day 14. Platelet levels declined during postoperative Day 1 and increased after Day 3, peaking between Days 7-14. Levels of $\beta$-thromboglobulin increased sharply and peaked at completion of surgery, followed by sustained release of $\beta$-thromboglobulin for 7 days and then a return to baseline levels. D-dimer concentrations also rose sharply at completion of surgery, declined at Day 3, and then increased by Day 7, remaining high until Day 14 (Table 2).

At completion of surgery, the following significant correlations between TEG values and blood/hemostatic parameters were noted: (1) $\mathrm{R}$ and $\beta$-thromboglobulin level $(\mathrm{r}=-0.54, \mathrm{p}<0.05)$; (2) Ang and $\beta$-thromboglobulin level $(\mathrm{r}=-0.65, \mathrm{p}<0.05)$; (3) MA and fibrinogen level $(\mathrm{r}=0.41, \mathrm{p}<0.05)$; (4) MA and platelet count $(\mathrm{r}=0.39, \mathrm{p}<0.05)$; (5) CI and platelet count $(\mathrm{r}=0.31, \mathrm{p}<0.05)$; and (6) $\mathrm{CI}$ and $\beta$-thromboglobulin level $(\mathrm{r}=0.60, \mathrm{p}<0.05)$.

On postoperative Day 1, CI correlated significantly with fibrinogen level $(\mathrm{r}=0.42, \mathrm{p}<0.05)$ and platelet count $(\mathrm{r}=0.36, \mathrm{p}<0.05)$.

By postoperative Day 7 , the following correlations were observed: (1) R and PT ( $r=0.48, \mathrm{p}<0.05)$; (2) Ang and PT $(\mathrm{r}=-0.45, \mathrm{p}<0.05)$; (3) CI and PT $(r=-0.45, p<0.05)$; and (4) Ly30 and fibrinogen level $(r=-0.41$, $\mathrm{p}<0.05)$.

By postoperative Day 14, the following determinants correlated significantly: (1) R and PT ( $\mathrm{r}=0.41, \mathrm{p}<0.05)$; (2) R and aPTT ( $\mathrm{r}=0.39$, $\mathrm{p}<0.05$; (3) Ang and PT ( $r=-0.51, \mathrm{p}<0.05)$; (4) Ang and aPTT $(\mathrm{r}=-0.46$, $\mathrm{p}<0.05)$; (5) MA and fibrinogen level $(\mathrm{r}=0.43, \mathrm{p}<0.05)$; (6) CI and PT $(\mathrm{r}=-0.47, \mathrm{p}<0.05)$; and 7$) \mathrm{CI}$ and aPTT $(\mathrm{r}=-0.46, \mathrm{p}<0.05)$.

\begin{tabular}{|c|c|c|c|c|c|c|}
\hline \multirow[b]{2}{*}{ Parameter } & \multicolumn{6}{|c|}{ Period concerning the operation } \\
\hline & Pre-THA & $30 \mathrm{~min}$ & $1^{\text {st }}$ day & $3^{\text {rd }}$ day & $7^{\text {th }}$ day & $14^{\text {th }}$ day \\
\hline $\mathrm{R}(\min )$ & $15.3(13.1-17.3)$ & $11.8(9.3-14.5)^{*}$ & $13.5(0.8-16.0)$ & $13.4(10.7-16.0)^{*}$ & $12.2(10.2-16.3)^{*}$ & $13.0(9.6-17.1)^{\star}$ \\
\hline Ang (deg) & $38.2(33.0-44.3)$ & $46.4(40.4-54.2)^{*}$ & $42.0(34.1-49.2)$ & $43.9(36.4-56.6)$ & $49.8(35.3-60.4)^{*}$ & $48.4(40.9-56.8)^{*}$ \\
\hline $\mathrm{MA}(\mathrm{mm})$ & $56.5(52.9-59.4)$ & $56.7(52.5-60.2)$ & $60.0(57.4-62.0)^{*}$ & $67.6(64.0-73.2)^{*}$ & $70.5(67.9-73.5)^{*}$ & $67.4(66.0-71.1)^{*}$ \\
\hline $\mathrm{Cl}$ & $-0.2(-1.0-0.7)$ & $0.2(-0.3-1.0)^{*}$ & $0.5(-0.2-1.1)^{*}$ & $1.9(1.1-2.6)^{*}$ & $2.3(1.6-3.0)^{*}$ & $1.8(1.3-2.7)^{*}$ \\
\hline Ly $30(\%)$ & $0.5(0.0-1.4)$ & $0.6(0.1-1.3)$ & $0.4(0.0-1.2)$ & $1.1(0.5-2.5)^{*}$ & $0.5(0.1-1.5)$ & $0.5(0.1-1.3)$ \\
\hline
\end{tabular}

Note: *Differences are significant as compared with the preoperative values $(p<0.05)$. Thromboelastography analysis $-\mathrm{R}$ : reaction time, Ang: alpha angle (the slope of the tracing, indicating the rapidity of fibrin build-up and cross-linking), MA: maximal amplitude, Cl: Coagulation Index, Ly 30: percent lysis 30 min after MA is reached.

Table 1: Coagulation and lysis in whole blood before and after hip arthroplasty. 


\begin{tabular}{|c|c|c|c|c|c|c|}
\hline \multirow[t]{2}{*}{ Parameter } & \multicolumn{6}{|c|}{ Period concerning the operation } \\
\hline & Pre-THA & $30 \mathrm{~min}$ & $1^{\text {st }}$ day & $3^{\text {rd }}$ day & $7^{\text {th }}$ day & $14^{\text {th }}$ day \\
\hline aPTT (s) & $28.8(26.5-31.3)$ & $28.5(25.8-32.2)$ & $29.3(26.8-32.8)^{*}$ & $28.0(26.2-30.9)$ & $28.2(26.3-30.4)$ & $28.6(27.3-32.4)$ \\
\hline PT (s) & $11.6(9.8-13.7)$ & $12.8(10.7-14.6)^{*}$ & $12.9(11.0-14.4)^{*}$ & $11.7(10.4-13.5)$ & $10.9(9.6-12.9)^{*}$ & $11.3(9.9-13.6)$ \\
\hline Fibrinogen (g/L) & $2.7(2.4-3.4)$ & $2.2(1.9-2.6)^{*}$ & $2.9(2.6-3.3)$ & $5.8(4.8-6.6)^{\star}$ & $5.2(4.4-5.9)^{*}$ & $4.2(3.8-4.9)^{*}$ \\
\hline TAT complexes $(\mu \mathrm{g} / \mathrm{L})$ & $4.7(1.7-6.8)$ & $28.0(17.2-29.4)^{*}$ & $11.1(8.3-18.2)^{*}$ & $6.2(5.0-7.5)$ & $5.8(5.4-7.2)$ & $6.1(4.3-7.8)$ \\
\hline D-dimer (ng/mL) & $97(42-196)$ & $1391(900-2264)^{*}$ & $978(682-2248)^{*}$ & $388(221-723)^{*}$ & $1113(710-1615)^{*}$ & $1003(660-1744)^{*}$ \\
\hline Platelets $\times 10^{9} / \mathrm{L}$ & $261(212-304)$ & $213(173-260)^{*}$ & $207(153-253)^{*}$ & $207(164-261)^{*}$ & $348(295-412)^{*}$ & $448(371-562)^{*}$ \\
\hline$\beta$-thromboglobulin (IUx109) & $115(78-148)$ & $293(161-455)^{*}$ & $161(115-404)^{*}$ & $153(118-326)^{*}$ & $108(75-221)$ & $93(64-116)$ \\
\hline
\end{tabular}

Note: *Differences are significant as compared with the preoperative values $(p<0.05)$

Table 2: Hemostasis parameters before and after hip arthroplasty.

\section{Discussion}

Major orthopedic surgery leads to massive tissue factor release and a hefty triggering of coagulation. Enzymatic reactions directed at thrombin production are greatly accelerated, intensifying TAT complex formation and consumption of coagulation factors as we and others have shown [15-20].

Ultimately, the time needed for latent-stage clot formation is significantly reduced. Although thrombin formation returns to baseline level by Day 3 after THA, TEG outcomes reveal that latency periods for clot formation remain below baseline determinations. Correlations between $\mathrm{R}$ value and other hemostatic measures of external and internal coagulation pathways throughout postoperative Weeks 1 and 2 are attributable to the influence of anticoagulant therapy.

Surgical intervention stimulates platelets to release granular factors [21]. Active secretion of $\beta$-thromboglobulin by platelets greatly impacts reaction time, rapidity of fibrin build-up, and overall coagulation potential, as confirmed by strong correlations between $\beta$-thromboglobulin level and TEG-measured clot formation. However, from postoperative Day 1 until the end of the study period, platelet activity had no bearing on clot formation in the blood.

After completion of THA, the rapidity of fibrin build-up and crosslinking was influenced by platelet activity. One week later, the Ang value primarily reflected activity of the external coagulation pathway; but on Day 14, both external and internal coagulation pathways were operant, apparently as an effect of anticoagulants.

Peak dynamic properties of fibrin and platelet bonding were evident beyond completion of surgery, despite a decline in concentration of fibrinogen and platelets that correlated with MA. This underscores the strength of GPIIb/IIIa receptors in fibrin/platelet binding. As in previous works [22-24], we observed an upsurge in MA after postoperative Day 1 , with high values persisting until the end of our study. During this period of time, fibrinogen and platelet concentrations clearly influenced MA, although direct correlations were not established.

The total coagulation potential of blood, expressed as integral CI, was increased after completion of arthroplasty, peaking at Day 7 and remaining elevated until the end of study. Of note, the major contributors to coagulation potential after completion of surgery were stimulated platelets and $\beta$-thromboglobulin activity. On postoperative Day 1, fibrinogen and platelet concentrations had the greatest effect on CI. From Day 7 to Day14, CI shows apparent correlations with PT and aPTT.. It may be that the relationship between total coagulation potential of blood and tests pertaining to external and internal coagulation pathways deviated on the basis of anticoagulant therapy.

In addition to substantial generation of localized fibrin, the trauma of surgery also promotes fibrinolysis. D-dimer, as a product of fibrin degradation, is subsequently increased [25]. Given the use of tranexamic acid, however, there was no evidence of an amplified lytic process in whole blood, as measured by TEG Ly30. By postoperative Day 3, the intensity of local fibrin degradation was reduced, in contrast to the lytic activity in whole blood, which was enhanced. One week after surgery, D-dimer levels indicated increasing fibrinolysis, which was sustained for at least the duration study. Active local fibrinolysis may contribute to the effective repair of vessels. At the same time, fibrinolysis in whole blood was returning to baseline level. No significant correlation between D-dimer levels and CI was evident during the study period.

In conclusion, although thrombin formation declines after major orthopedic surgery, the total coagulation potential of blood (under standard anticoagulant treatment) peaks in one week and remains elevated at least until postoperative Day 14, correlating with external coagulation pathway activity during this time. The fibrinolytic activity of whole blood is unchanged during the postoperative period, with the exception of an increase on postoperative Day 3. It is our contention that use of TEG for monitoring coagulation and thromboembolic prophylaxis following major orthopedic surgery merits further investigation.

\section{References}

1. Yukizawa $\mathrm{Y}$, Inaba $\mathrm{Y}$, Watanabe S, Yajima S, Kobayashi N, et al. (2012 Association between venous thromboembolism and plasma levels of both soluble fibrin and plasminogen-activator inhibitor 1 in 170 patients undergoing total hip arthroplasty. Acta Orthop 83: 14-21.

2. Lowe GD (1997) Prediction of postoperative venous thrombosis using haemostasis tests. Int J Clin Lab Res 27: 153-157.

3. Rosendaal FR (1999) Venous thrombosis: a multicausal disease. Lancet 353 : $1167-1173$

4. Bick RL, Kaplan H (1998) Syndromes of thrombosis and hypercoagulability. Congenital and acquired causes of thrombosis. Med Clin North Am 82: 409458.

5. Craven S, Dewar L, Yang X, Ginsberg J, Ofosu F (2007) Altered regulation of in-vivo coagulation in orthopedic patients prior to knee or hip replacement surgery. Blood Coagul Fibrinolysis 18: 219-225.

6. Geerts WH, Bergqvist D, Pineo GF, Heit JA, Samama CM, et al. (2008) American College of Chest Physicians. Prevention of venous thromboembolism: American College of Chest Physicians Evidence-Based Clinical Practice Guidelines. Chest 133: 381-453.

7. Lanevschi A, Kramer JW, Greene SA, Meyers KM (1996) Fibrinolytic activity in dogs after surgically induced trauma. Am J Vet Res 57: 1137-1140.

8. Kageyama K, Nakajima Y, Shibasaki M, Hashimoto S, Mizobe T (2007) Increased platelet, leukocyte, and endothelial cell activity are associated with increased coagulability in patients after total knee arthroplasty. J Thromb Haemost 5: 738-745.

9. Reikerås $\mathrm{O}$, Clementsen $\mathrm{T}$ (2009) Time course of thrombosis and fibrinolysis in total knee arthroplasty with tourniquet application. Local versus systemic activations. J Thromb Thrombolysis 28: 425-428. 
Citation: Antropova IP, Reino EV, Yushkov BG, Shlykov IL, Varaksin AN (2013) Blood Coagulation at Major Orthopaedic Surgery. J Blood Disorders Transf 5: 178. doi: 10.4172/2155-9864.1000178

Page 4 of 4

10. Brueckner S, Reinke U, Roth-Isigkeit A, Eleftheriadis S, Schmucker P, et al. (2003) Comparison of general and spinal anesthesia and their influence on hemostatic markers in patients undergoing total hip arthroplasty. J Clin Anesth 15: 433-440.

11. Whitten CW, Greilich PE (2000) Thromboelastography: past, present, and future. Anesthesiology 92: 1223-1225.

12. Favaloro EJ, Lippi G (2011) Coagulation update: what's new in hemostasis testing? Thromb Res 127: S13-16.

13. Handa A, Cox DJ, Pasi KJ (1998) Thromboelastography: an effective screening test for prothrombotic states. Br J Surg 85: 90

14. Kashuk JL, Moore EE, Sabel A, Barnett C, Haenel J, et al. (2009) Rapid thrombelastography ( $r-T E G)$ identifies hypercoagulability and predicts thromboembolic events in surgical patients. Surgery 146: 764-772.

15. Rosencher N, Vassilieff N, Guigonis V, Toulon P, Conseiller C (1992) [Comparison of effects of Elohes and albumin on hemostasis in orthopedic surgery]. Ann Fr Anesth Reanim 11: 526-530.

16. Wei KL, Lin CJ, Lai KA (1995) Changes in coagulatory profile after orthopedic surgery. J Formos Med Assoc 94: 541-547.

17. Høgevold HE, Lyberg T, Kähler H, Reikerås O (1996) Expression of beta-2integrins and L-selectin by leukocytes and changes in acute-phase reactants in total hip replacement surgery. Eur Surg Res 28: 190-200.

18. Sokolowska B, Piecuch W, Walter-Croneck A, Dmoszynska A, Furmanik $F$ (2002) Evaluation of selected parameters of blood coagulation and the fibrinolysis system in patients undergoing total hip replacement. Przegl Lek 59: 502-508.

19. Fedi S, Gori AM, Falciani M, Cellai AP, Aglietti P, et al. (1999) Proceduredependence and tissue factor-independence of hypercoagulability during orthopaedic surgery. Thromb Haemost 81: 874-878.

20. López Y, Páramo JA, Valentí JR, Pardo F, Montes R, et al. (1997) Hemostatic markers in surgery: a different fibrinolytic activity may be of pathophysiological significance in orthopedic versus abdominal surgery. Int J Clin Lab Res 27: 233-237.

21. Bunescu A, Widman J, Lenkei R, Menyes P, Levin K, et al. (2002) Increases in circulating levels of monocyte-platelet and neutrophil-platelet complexes following hip arthroplasty. Clin Sci (Lond) 102: 279-286.

22. Mahla E, Lang T, Vicenzi MN, Werkgartner G, Maier R, et al. (2001) Thromboelastography for monitoring prolonged hypercoagulability after major abdominal surgery. Anesth Analg 92: 572-577.

23. Wilson D, Cooke EA, McNally MA, Wilson HK, Yeates A, et al. (2001) Changes in coagulability as measured by thrombelastography following surgery for proximal femoral fracture. Injury 32: 765-770.

24. Gibbs NM, Bell R (1998) The effect of low-dose heparin on hypercoagulability following abdominal aortic surgery. Anaesth Intensive Care 26: 503-508.

25. Pape HC, Schmidt RE, Rice J, van Griensven M, das Gupta R, et al. (2000) Biochemical changes after trauma and skeletal surgery of the lower extremity: quantification of the operative burden. Crit Care Med 28: 3441-3448. 